Check for updates

Cite this: Phys. Chem. Chem. Phys., 2018, 20, 5246

Received 16th October 2017 Accepted 19th January 2018

DOI: $10.1039 / c 7 c p 07052 g$

rsc.li/pccp

\section{Solvation dynamics in polar solvents and imidazolium ionic liquids: failure of linear response approximations $\dagger$}

\begin{abstract}
Esther Heid (D) and Christian Schröder (D) *
This study presents the large scale computer simulations of two common fluorophores, $\mathrm{N}$-methyl-6oxyquinolinium betaine and coumarin 153, in five polar or ionic solvents. The validity of linear response approximations to calculate the time-dependent Stokes shift is evaluated in each system. In most studied systems linear response theory fails. In ionic liquids the magnitude of the overall response is largely overestimated, and linear response theory is not able to capture the individual contributions of cations and anions. In polar liquids, the timescales of solvation dynamics are often not correctly reproduced. These observations are complemented by a detailed analysis of Gaussian statistics including higher order correlation functions, variance of the energy gap distribution and its time evolution. The analysis of higher order correlation functions was found to be not suitable to predict a failure of linear response theory. Further analysis of radial distribution functions and hydrogen bonds in the ground and excited state, as well as the time evolution of the number of hydrogen bonds after solute excitation reveal an influence of solvent structure in some of the studied systems.
\end{abstract}

\section{Introduction}

The time-dependent Stokes shift (TDSS) describes the rate of solvent reorganization after an electric perturbation inflicted by the excitation of an immersed chromophore. The TDSS has been extensively used to investigate the solvation dynamics of polar solvents over the last decades. ${ }^{1-16}$ With the development of ionic liquids as interesting new solvents for diverse reactions, the TDSS has also become a powerful tool to examine the electrostatic solvation in ionic, non-aqueous media. ${ }^{17-29}$ In contrast to conventional solvents such as water and alcohols, the solvation response in ionic liquids is extremely slow, as it scales with viscosity. ${ }^{25}$ A number of computer simulations have been conducted to examine the interesting solvation behavior of ionic liquids..$^{22,25,30-32}$ It was shown that the solvation energies of ionic liquids resemble those in conventional solvents and most of the solvation energy stems from anion movement. However, the computer simulation of solvation dynamics is often realized via invocation of linear response theory (LRT), which may not be valid for arbitrary solute-solvent combinations. Within linear response theory, the true solvent reorganization

University of Vienna, Faculty of Chemistry, Department of Computational Biological Chemistry, Währingerstraße 19, A-1090 Vienna, Austria.

E-mail: christian.schroeder@univie.ac.at; Tel: +43 1427752711

$\dagger$ Electronic supplementary information (ESI) available: Higher order and partial correlation functions, corrections to Gaussian statistics and structure analysis for all systems. See DOI: 10.1039/c7cp07052g after the excitation of a solute is resembled by the extrapolation of the energy gap fluctuations between the ground and excited state. This approximation may save a lot of computation time, but at the cost of being valid only in systems where either the perturbation is small, or the energy gap fluctuations are Gaussian. ${ }^{4,33-35}$ We recently showed that LRT fails for systems where the energy gap is locally Gaussian, but non-stationary (changes with time), or if large changes in solvent structure occur after perturbation, both leading to a globally non-gaussian distribution. ${ }^{36}$

In literature, the TDSS is often calculated only via LRT $^{27,30,37-46}$ and the true nonequilibrium response of a system remains unknown. This is especially true if computationally expensive all-atomic and polarizable ionic liquid forcefields are used. Ref. 32 (or the earlier work ref. 31) is frequently used to justify the use of linear response theory in solvation dynamics of ionic liquids. ${ }^{30,37,39-43}$ In this work of Kim and coworkers a set of 400 nonequilibrium simulations extending to $2 \mathrm{ps}$ is used to describe the solvation dynamics of artificial solutes in two imidazolium ionic liquids, and compared to equilibrium simulations invoking linear response theory. ${ }^{32}$ Although the authors argue that linear response holds reasonable well in their system, the agreement between the Stokes shift from their equilibrium and nonequilibrium simulations seems to be unsatisfactory, and the integral timescales differ considerably. Furthermore, ionic liquid dynamics, extending to nanoseconds, cannot be simulated accurately on such a short timescale. Although this work comprises valuable insights into ionic liquid solvation, it cannot 
be invoked to justify the choice of LRT for solvation dynamics in ionic liquids without further verification. A more recent study of Maroncelli and coworkers revealed the failure of linear response for the solvation dynamics of small solutes in ionic liquids. ${ }^{25}$ Current advances in technology opened up the possibility of calculating large scale nonequilibrium simulations, so that the validity of LRT in these systems can now directly be assessed. This study therefore focuses on the validity of the linear response approximation both in polar and ionic liquids using fully atomistic, flexible and polarizable force fields by direct comparison to nonequilibrium simulations, as well as experimental data. Real chromophores are employed as solutes, as the solvation response depends strongly on the nature of solute. Thus, the commonly used artificial solutes can only give general information. We study $N$-methyl-6-oxyquinolinium betaine (MQ) and coumarin 153 (C153) as the former lowers its dipole moment upon excitation, whereas the latter strengthens it, so that the validity of linear response can be tested for both an increase and decrease of the dipole moment during excitation. Furthermore the ability of partial correlation functions to depict cationic and anionic contributions is assessed. Previously, different findings were reported on the predominant role of cations, ${ }^{47}$ anions $^{32}$ or both ${ }^{30,48,49}$ for the short-time response, where some of the conclusions were drawn from equilibrium simulations. These results are complemented by an analysis of higher-order correlation functions and their ability to predict LRT validity, ${ }^{35,50}$ solvent structure via radial distribution functions and coordination numbers, as well as a time-evolution of the spectral width. Therefore, this work gives valuable insight into when and why linear response is applicable in real systems.

\section{Theory}

The following equations only comprise the most important relations of linear response theory, which will be employed in this study. The derivation of these relations, as well as a more detailed description, can be found in literature. ${ }^{4,33-36}$

Experimentally, a fluorescent molecular probe is introduced to the solvent of interest, excited by a laser beam, and its subsequent time-dependent change in fluorescence frequency monitored. The Stokes shift relaxation function can then be calculated as

$$
S(t)=\frac{h \nu(t)-h \nu(\infty)}{h \nu(0)-h \nu(\infty)}
$$

which is simply the normalized change in energy. Computationally, $S(t)$ can be evaluated as the average change in interaction energy $\Delta U$

$$
S(t)=\frac{\overline{\Delta U(t)}-\overline{\Delta U(\infty)}}{\overline{\Delta U(0)}-\overline{\Delta U(\infty)}}
$$

after excitation of a suitable chromophore. This procedure requires the actual excitation of the solute, so that an excited state force field is needed, as well as averaging over hundreds of different conformations. To avoid this computationally expensive method, often linear response theory (LRT) is invoked instead. Conventional
LRT links the energy fluctuations $\delta \Delta U(t)=\Delta U(t)-\langle\Delta U\rangle$ on the unperturbed energy surface (the equilibrated ground state) to $S(t)$ via

$$
S(t) \simeq \frac{\langle\delta \Delta U(0) \delta \Delta U(t)\rangle_{\mathrm{g}}}{\left\langle\delta \Delta U(0)^{2}\right\rangle_{\mathrm{g}}}=C_{\mathrm{g}}(t)
$$

where we assume that the perturbation is small on a scale of $k_{\mathrm{B}} T$. Alternatively, the energy fluctuations on the perturbed energy surface (the equilibrated excited state) can be linked to $S(t)$ via

$$
S(t) \simeq \frac{\langle\delta \Delta U(0) \delta \Delta U(t)\rangle_{\mathrm{e}}}{\left\langle\delta \Delta U(0)^{2}\right\rangle_{\mathrm{e}}}=C_{\mathrm{e}}(t)
$$

In both cases, the large number of nonequilibrium simulations needed for eqn (2) are replaced by a single equilibrium simulation on the ground or excited state. The latter relation is true even for large perturbations, if the system exhibits Gaussian statistics. ${ }^{34,35}$ Then, higher order correlation functions which are treated as zero in conventional LRT, are taken into account, as they are multiples of $\langle\delta \Delta U(0) \delta \Delta U(t)\rangle_{\mathrm{e}}$ (odd-numbered), or zero (even-numbered): ${ }^{51}$

$$
\begin{aligned}
\left\langle\delta \Delta U(0)^{2 n+1} \delta \Delta U(t)\right\rangle_{\mathrm{e}}= & \frac{(2 n+1) !}{n ! 2^{n}}\langle\delta \Delta U(0) \delta \Delta U(t)\rangle_{\mathrm{e}} \\
& \cdot\left\langle\delta \Delta U(0)^{2}\right\rangle_{\mathrm{e}}^{n}
\end{aligned}
$$

The Stokes shift relaxation function is thus (again) described by eqn (4), but on different mathematical grounds. The absolute Stokes shift $\Delta \Delta U$, which is $\Delta U(0)-\Delta U(\infty)$ is then

$$
\Delta \Delta U \simeq \frac{1}{k_{\mathrm{B}} T}\left\langle\delta \Delta U(0)^{2}\right\rangle_{\mathrm{e}}=\frac{W^{2}}{k_{\mathrm{B}} T}
$$

in the linear response approximation, with the spectral width

$$
W=\sqrt{\overline{\Delta U^{2}}-(\overline{\Delta U})^{2}}
$$

\section{Methods}

Equilibrium simulations in the ground and excited state, as well as nonequilibrium simulations of the solutes coumarin 153 (C153) and $N$-methyl-6-oxyquinolinium betaine (MQ) were carried out in the polar solvents acetonitrile (ACN), methanol $(\mathrm{MeOH})$ and 2-propanol (2-PrOH), as well as in the ionic liquids 1-ethyl-3-methylimidazolium dicyanamide ([ $\left.\left.\operatorname{Im}_{21}\right][\mathrm{DCA}]\right)$ and 1-ethyl-3-methylimidazolium trifluoromethanesulfonate $\left(\left[\operatorname{Im}_{21}\right][\mathrm{OTf}]\right)$. The solvents were chosen to cover a wide range of viscosities, as the timescale of the time-dependent Stokes shift, and therefore its range, depends directly on solvent viscosity. ${ }^{25}$ All simulations were conducted with the program package CHARMM. ${ }^{52}$ The force field for MQ was taken from ref. 53. The force field for C153 was set up accordingly, namely the geometry was optimized at the B3LYP $6-311 \mathrm{G}++(2 \mathrm{~d}, 2 \mathrm{p})$ level of theory using density functional theory (DFT) and the respective values put into the intramolecular potentials obtained from PARAMCHEM ${ }^{54,55}$ and the CHARMM General Force Field (CGenFF). ${ }^{56}$ The partial charges were calculated using TD-DFT with the $\omega \mathrm{B} 97 \mathrm{xD}$ hybrid DFT functional $^{57}$ and the CHelpG method. ${ }^{58}$ 
The force fields of the ionic liquids were taken from Padua and coworkers, ${ }^{59-61}$ and made polarizable with atomic polarizabilities from ref. 62. Polarizability is especially important to correctly depict dynamic properties of ionic liquids. For $\mathrm{MeOH}$ and 2-PrOH, polarizable force fields were taken from ref. 63 and the CGenFF. For ACN, a suitable nonpolarizable force field was taken from the CGenFF. We note that for polar liquids, polarizable force fields are not always superior to well tuned nonpolarizable forcefields (see also ref. 53), so that this choice should not affect the outcome of this study. All trajectories were calculated using the Velocity-Verlet integrator with a timestep of 1 fs (0.5 fs during NPT simulation) and a Nosé-Hoover thermostat. ${ }^{64,65}$ Periodic boundary conditions were used, where electrostatic interactions were calculated using the particle mesh Ewald method (grid size $1 \AA$, cubic splines of order 6 , a $\kappa$ of $0.41 \AA^{-1}$ ) and van der Waals interactions were cut off at $12 \AA$.

All systems were randomly packed to cubic simulation boxes using PACKMOL ${ }^{66}$ and converged during a subsequent $0.5 \mathrm{~ns}$ $N P T$ equilibration to the boxlengths listed in Table 1 . The equilibrium simulations were conducted at $300 \mathrm{~K}$ after an equilibration of 1 to 5 ns for the time periods listed in Table 1 . Nonequilibrium simulations were calculated using 500 independent starting configurations obtained from either long $N V T$ simulations or independently packed boxes. Where necessary, the starting configurations were further equilibrated for $0.5 \mathrm{~ns}$ for the polar solvents, and $1 \mathrm{~ns}$ for the ionic liquids per replica. The trajectories were then monitored after sudden change of the partial charge distribution from ground to excited state for time periods according to Table 1 . Thus, a total of $4.2 \mu \mathrm{s}$ of fully polarizable, atomic molecular dynamics simulation was produced in this study, not counting in another few microseconds of equilibration of starting configurations. To the best of our knowledge, no simulation study of the Stokes shift exists on such a large scale. The resulting trajectories were analyzed via a python program based on MDAnalysis. ${ }^{67} 95 \%$ confidence intervals are given, where appropriate, as $\bar{x} \pm \frac{t \cdot s}{\sqrt{n}}$ where $\bar{x}$ is the average of the property of interest, $t$ the Student $t$ factor, $s$ the standard deviation and $n$ the number of independent trajectories. For the nonequilibrium simulations, $n$ is simply the number of simulated trajectories; for the equilibrium simulation the long trajectory was cut into 5 parts and analyzed separately to yield an upper bound of the true confidence interval.

Table 1 Boxlengths of cubic simulation boxes and lengths of simulation of equilibrium (EQ) and nonequilibrium (NEQ) trajectories

\begin{tabular}{llll}
\hline Content & Boxlength $(\AA)$ & EQ $(\mathrm{ns})$ & NEQ \\
\hline $1 \mathrm{MQ}+1000 \mathrm{ACN}$ & 44.40 & 20 & $500 \times 100 \mathrm{ps}$ \\
$1 \mathrm{C} 153+1000 \mathrm{ACN}$ & 44.40 & 20 & $500 \times 100 \mathrm{ps}$ \\
$1 \mathrm{MQ}+1000 \mathrm{MeoH}$ & 40.45 & 20 & $500 \times 100 \mathrm{ps}$ \\
$1 \mathrm{C} 153+1000 \mathrm{MeOH}$ & 40.47 & 20 & $500 \times 100 \mathrm{ps}$ \\
$1 \mathrm{MQ}+10002-\mathrm{ProH}$ & 50.61 & 20 & $500 \times 100 \mathrm{ps}$ \\
$1 \mathrm{C} 153+10002-\mathrm{PrOH}$ & 50.61 & 20 & $500 \times 100 \mathrm{ps}$ \\
$1 \mathrm{MQ}+500\left[\mathrm{Im}_{21}\right][\mathrm{DCA}]$ & 51.17 & 50 & $500 \times 1 \mathrm{~ns}$ \\
$1 \mathrm{C} 153+500\left[\mathrm{Im}_{21}\right][\mathrm{DCA}]$ & 51.15 & 50 & $500 \times 1 \mathrm{~ns}$ \\
$1 \mathrm{MQ}+500\left[\mathrm{Im}_{21}\right][\mathrm{OTf}]$ & 53.52 & 100 & $500 \times 2.5 \mathrm{~ns}$ \\
$1 \mathrm{C} 153+500\left[\mathrm{Im}_{21}\right][\mathrm{OTf}]$ & 53.45 & 100 & $500 \times 2.5 \mathrm{~ns}$
\end{tabular}

\section{Results and discussion}

\subsection{Comparison of equilibrium and nonequilibrium relaxation functions and absolute shifts}

The Stokes shift relaxation function $S(t)$ from non-equilibrium simulations, as well as the respective time correlation functions $C_{\mathrm{g}}(t)$ and $C_{\mathrm{e}}(t)$ from equilibrium simulations are shown in Fig. 1 for polar solvents and in Fig. 2 for ionic solvents. A linear-linear plot (instead of the logarithmic scale of the time axis in Fig. 1) for polar solvents, as well as a plot of the logarithm of the Stokes shift for all solvents can be found in the ESI. $\dagger$

If conventional linear response theory is applicable, $C_{\mathrm{g}}(t)$, $C_{\mathrm{e}}(t)$ and $S(t)$ should correspond well to each other. This is only true for ACN as solvent. For all other solvents and for both chromophores, the full relaxation curves from equilibrium and nonequilibrium simulations do not match. Especially in the case of ionic liquids LRT underestimates the amount of early relaxation. The insets in Fig. 1 and 2 show the relaxation after $0.1 \mathrm{ps}$, where the curves were set to 1 at $0.1 \mathrm{ps}$, as experimental data shown here is mostly normalized at this point in time, too. For MQ, this normalization does not lead to a good agreement of $C_{\mathrm{g}}(t)$ or $C_{\mathrm{e}}(t)$ with the true $S(t)$ curves, neither in $\mathrm{MeOH}$ or 2-PrOH, nor in the ionic liquids $\left[\mathrm{Im}_{21}\right][\mathrm{DCA}]$ or $\left[\mathrm{Im}_{21}\right][\mathrm{OTf}]$, although in the latter the discrepancy is very small. For the

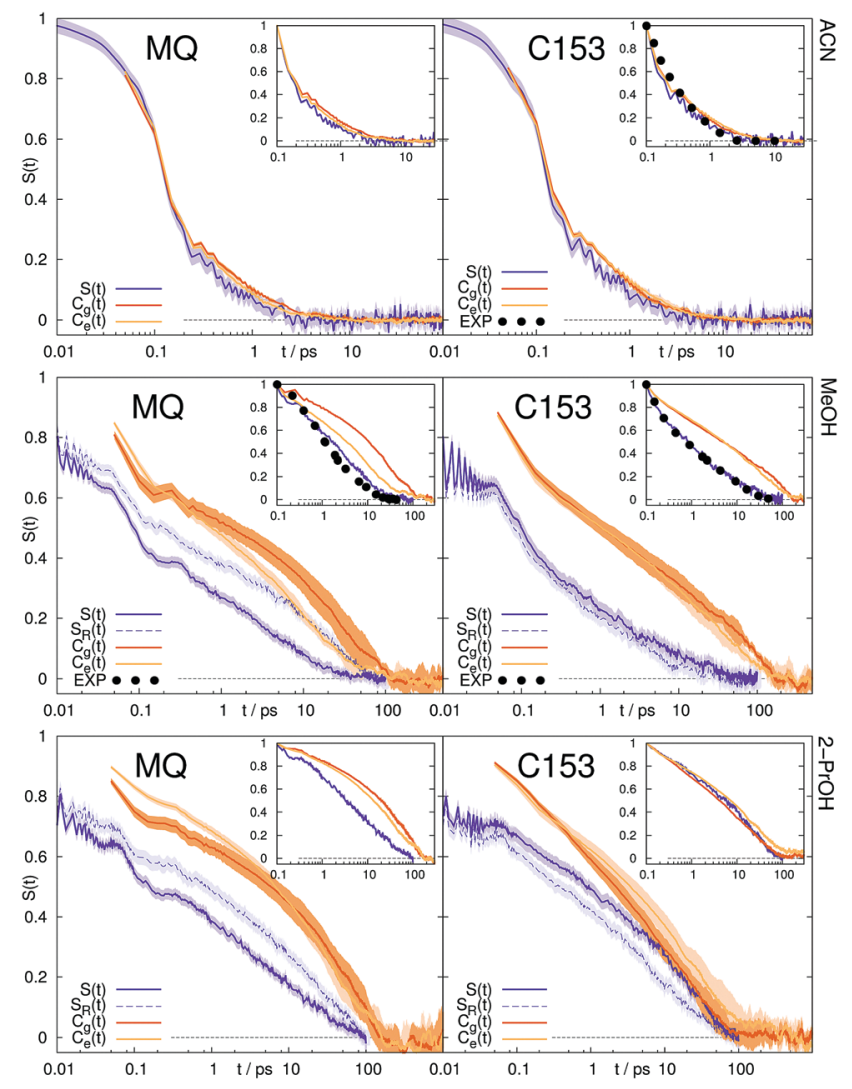

Fig. 1 Stokes shift relaxation function after excitation, $S(t)$, after deexcitation, $S_{R}(t)$, and time correlation functions $C_{g}(t)$ and $C_{e}(t)$ in ground and excited state in acetonitrile (top), methanol (middle) and 2-propanol (bottom) for MQ (left) and C153 (right). The colored area corresponds to a $95 \%$ confidence interval. Experimental data (labeled EXP) are taken from ref. 14 and 68. 

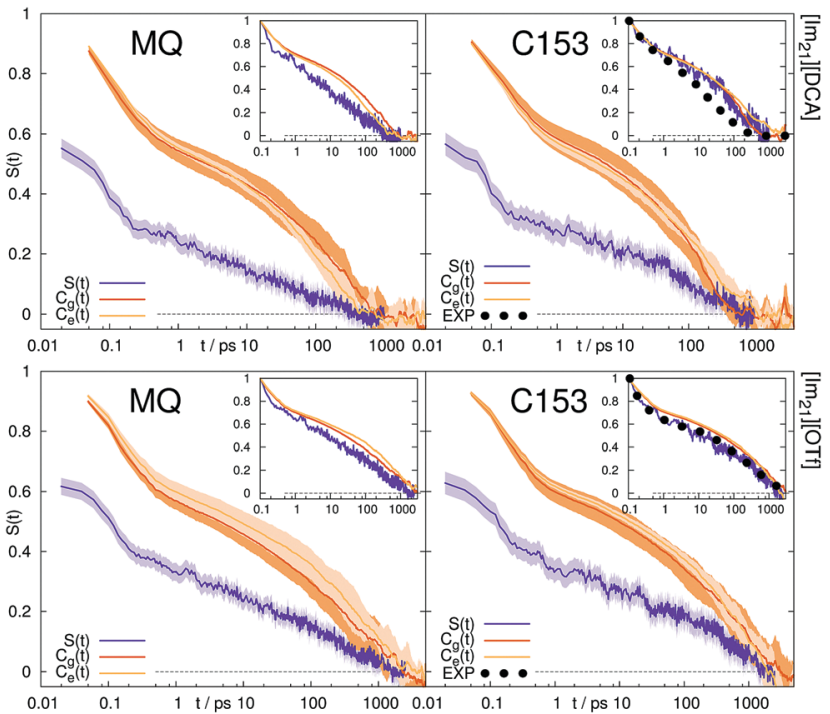

Fig. 2 Stokes shift relaxation function $S(t)$ and time correlation functions $C_{g}(t)$ and $C_{e}(t)$ in ground and excited state in $\left[m_{21}\right][D C A]$ (top) and $\left[\mathrm{m}_{21}\right][\mathrm{OTf}]$ (bottom) for MQ (left) and C153 (right). The colored area corresponds to a $95 \%$ confidence interval. Experimental data (labeled EXP) are taken from ref. 69

chromophore C153 a different picture arises: The linear response approximation seems to hold after $0.1 \mathrm{ps}$ for the solvents 2-PrOH, $\left[\mathrm{Im}_{21}\right][\mathrm{DCA}]$ and $\left[\mathrm{Im}_{21}\right][\mathrm{OTf}]$ and only fails in $\mathrm{MeOH}$, for which a failure of linear response is already known. ${ }^{70-72}$ An early study of $\mathrm{C} 153$ in ACN and $\mathrm{MeOH}$ finds contradictory results, namely that linear response theory holds. ${ }^{73}$ However, due to the limited computer power back then, the equilibrium simulations are not long enough to yield statistically significant results for $\mathrm{MeOH}$, so that we attribute our different finding to better sampling. Incidentally, the different solvent force fields contribute to the dissimilar conclusions drawn, too. The effect of sampling and force field in our study, as well as in the system of ref. 73 is given in the ESI. $\dagger$ For C153 in 2-PrOH, $\left[\operatorname{Im}_{21}\right][\mathrm{DCA}]$ and $\left[\mathrm{Im}_{21}\right][\mathrm{OTf}]$ the curvature at later times is depicted correctly, although the overall response does not depict $S(t)$. Thus, LRT seems to be applicable for most simulations of $\mathrm{C} 153$ for the non-inertial solvation response. The inertial solvation response, however, cannot be described via LRT for both chromophores in all studied solvents but ACN. The insets in Fig. 1 and 2 furthermore show the experimentally obtained Stokes shift relaxation functions, ${ }^{14,68,69}$ which correspond very well to the calculated $S(t)$ throughout all systems.

To compare the different timescales of solvation, it is convenient to calculate the integral relaxation time

$$
\tau=\int_{0}^{\infty} S(t) \mathrm{d} t
$$

(analogous definition for $C_{\mathrm{g}}(t)$ and $\left.C_{\mathrm{e}}(t)\right)$. The relaxation times are listed in Table 2 . The relaxation times obtained from nonequilibrium simulations follow the expected trends, namely show an increase of relaxation time with an increase in viscosity of the solvent. Furthermore, the relaxation times of MQ are
Table 2 Relaxation times $\tau$ of the Stokes shift relaxation function from nonequilibrium $(S(t))$ and equilibrium simulations in the ground $\left(C_{g}(t)\right)$ and excited state $\left(C_{e}(t)\right)$. Experimental viscosities ${ }^{74-77}$ at $298.15 \mathrm{~K}$

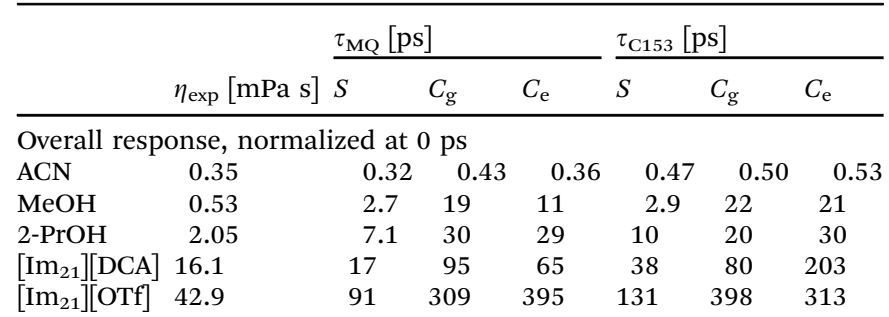

Response in the diffusive regime, normalized at $0.1 \mathrm{ps}$ $\begin{array}{llllllll}\text { ACN } & 0.35 & 0.33 & 0.62 & 0.48 & 0.47 & 0.67 & 0.79\end{array}$ $\begin{array}{llllllll}\mathrm{MeOH} & 0.53 & 5.8 & 29 & 16 & 5.8 & 30 & 28\end{array}$ $\begin{array}{llllllll}2-\mathrm{PrOH} & 2.05 & 13 & 40 & 34 & 15 & 15 & 26\end{array}$

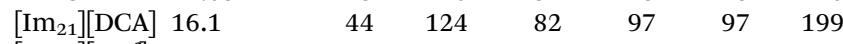

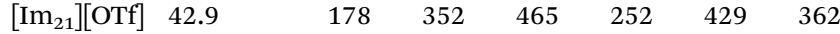

always less than for the larger chromophore C153, which is expected from the correlation of solute rotation (and thus its volume) and relaxation time in slowly rotating solvents. To visualize this behavior, we plotted the relaxation time against the solvent viscosity, shown in Fig. 3. As mentioned, the relaxation time increases with the viscosity $\eta_{\text {exp }}$, MQ relaxes faster than C153 and the difference between them becomes larger as the correlation of solute and solvent rotation increases. The relaxation times obtained from equilibrium simulations, however, raise a number of problems. First, they overestimate the timescale of solvation by a factor of two to eight. Second, they do not scale correctly with viscosity, for example $\mathrm{C} 153$ in 2-PrOH relaxes faster than in $\mathrm{MeOH}$. Third, MQ relaxes not always faster than C153, so that the lines in Fig. 3 sometimes decrease or intersect each other. Thus, the absolute timescales provided by equilibrium simulations are not an accurate reflection of reality.

Since we pointed out earlier that the inertial relaxation is not described correctly via LRT in all solvents but ACN, timescales of the relaxation after $0.1 \mathrm{ps}$ are also given in Table 2 . They are calculated as the integral of $S(t)$ normalized at $0.1 \mathrm{ps}$ (i.e. the area under the curve depicted in the insets in Fig. 1 and 2). The obtained relaxation times describe the timescale of diffusive contributions and collective rotation. ${ }^{78,79}$ Yet again the same problems arise, namely the use of LRT falsely leads to the assumption that the excited state C153 is solvated faster in

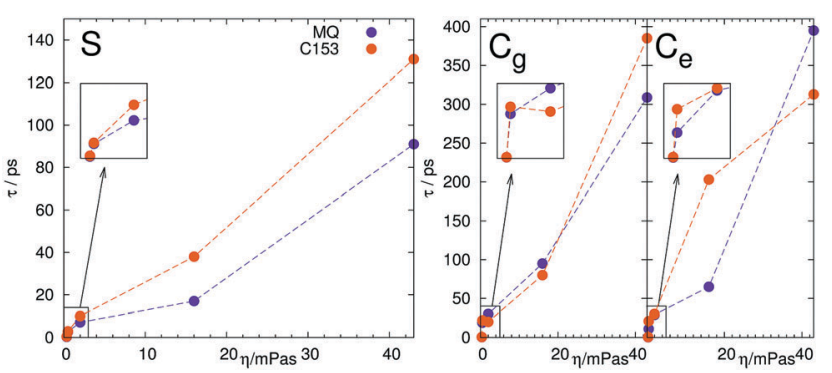

Fig. 3 Scaling of the relaxation time $\tau$ from nonequilibrium $(S(t)$, left) and equilibrium simulations in the ground $\left(C_{g}(t)\right.$, middle) and excited state $\left(C_{\mathrm{e}}(t)\right.$, right) with solvent viscosity $\eta_{\exp }$ (see Table 2$)$. 
Table 3 Absolute Stokes shift $\Delta \Delta U$ in $\mathrm{kJ} \mathrm{mol}^{-1}$ obtained from nonequilibrium simulations $(S(t))$ and equilibrium simulations in the ground $\left(C_{\mathrm{g}}(t)\right)$ and excited $\left(C_{\mathrm{e}}(t)\right)$ state, as well as experimental Stokes shift via $h[\nu(\infty)-\nu(0)]$ from ref. 14 and 69

\begin{tabular}{llclll}
\hline System & & $\Delta \Delta U_{C_{\mathrm{g}}}$ & $\Delta \Delta U_{C_{\mathrm{e}}}$ & $\Delta \Delta U_{\mathrm{S}}$ & $\Delta \Delta U_{\text {exp }}$ \\
\hline MQ & ACN & 27 & 30 & 27 & \\
& MeOH & 52 & 48 & 52 & 42 \\
& $2-\mathrm{PrOH}$ & 44 & 46 & 44 & \\
& {$\left[\mathrm{Im}_{21}\right][\mathrm{DCA}]$} & 77 & 79 & 38 & \\
& {$\left[\mathrm{Im}_{21}\right][\mathrm{OTf}]$} & 67 & 72 & 38 & \\
$\mathrm{C} 153$ & ACN & 32 & 30 & 27 & 27 \\
& MeOH & 40 & 39 & 35 & 37 \\
& $2-\mathrm{PrOH}$ & 21 & 25 & 28 & \\
& {$\left[\mathrm{Im}_{21}\right][\mathrm{DCA}]$} & 101 & 96 & 36 & 25 \\
& {$\left[\mathrm{Im}_{21}\right][\mathrm{OTf}]$} & 81 & 83 & 33 & 28 \\
& & & & & \\
& & & &
\end{tabular}

2-PrOH than in MeOH. Similarly, LRT claims that MQ in 2-PrOH or $\left[\mathrm{Im}_{21}\right][\mathrm{DCA}]$ is solvated on a slower timescale than C153, which is not observed from nonequilibrium simulations.

Apart from the timescales discussed above, also the absolute Stokes shift $\Delta \Delta U$ obtained from equilibrium and non-equilibrium simulations should be identical if a system exhibits a linear solvation response. Table 3 lists the absolute shifts obtained from non-equilibrium and equilibrium simulations, as well as experimental absolute shifts from ref. 14 and 69. The shifts $\Delta \Delta U$ were calculated as $h[\nu(0)-\nu(\infty)]$ from experiment, as $\Delta U(0)-\Delta U(\infty)$ from nonequilibrium simulations and via eqn (6) from equilibrium simulations. For the polar solvents, LRT reproduces the absolute shifts from nonequilibrium simulations and experiment, even where the curvature of the LRT Stokes shift relaxation function is quantitatively wrong (e.g. for $1 \mathrm{MQ}$ or $\mathrm{C} 153$ in $\mathrm{MeOH})$. For the ionic liquids, the linear response approximation overestimates the absolute shifts by a factor of 2 to 3 .

Thus, the validity of LRT even for coumarin 153 in ionic liquids is not confirmed, in contrast to the general findings in ref. 32. The implications of the overestimation of the overall shift by the time correlation functions become also visible when the Stokes shift relaxation function is plotted unnormalized, as shown in Fig. 4 for $\mathrm{C} 153$ in $\mathrm{MeOH}$ and [ $\left.\mathrm{Im}_{21}\right][\mathrm{OTf}]$. In MeOH, LRT correctly reproduces the absolute shift, so that both the normalized and unnormalized relaxation functions show the same trend. Again, the nonequilibrium simulations fit very well to experimental data,

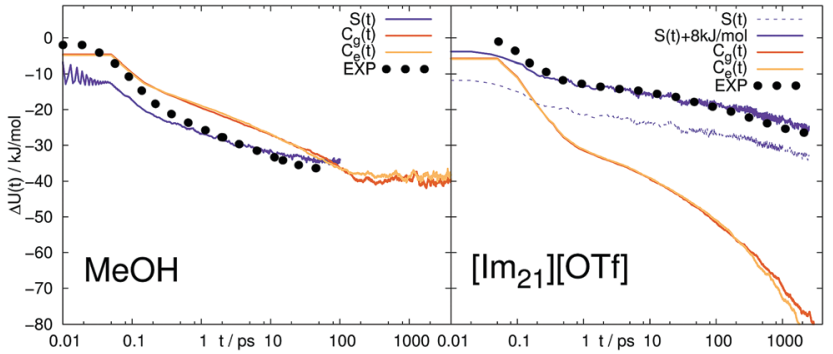

Fig. 4 Absolute change in energy $\Delta U(t)$ calculated from the Stokes shift relaxation function $S(t)$ and time correlation functions $C_{g}(t)$ and $C_{e}(t)$ in ground and excited state in $\mathrm{MeOH}$ (left) and [lm $\left.\mathrm{lm}_{21}\right]$ [OTf] (right) for $\mathrm{C} 153$. Experimental data (labeled EXP) are taken from ref. 14 and 69. which is remarkable given the fact that the curves are not normalized and were not set to the same value at a specific point. In $\left[\mathrm{Im}_{21}\right][\mathrm{OTf}]$, the relaxation functions obtained via LRT does not describe neither the nonequilibrium counterpart nor experiment. Note that $S(t)$ is plotted twice, once set to 0 at $0 \mathrm{fs}$, and once at $14 \mathrm{fs}$, which results in a constant offset of about $8 \mathrm{~kJ} \mathrm{~mol}^{-1}$. As the experimental resolution is about $80 \mathrm{fs}$, and the value at time 0 is only estimated, both representations of $S(t)$ are equally valid and produce a very good fit to experiment.

The magnitude of the absolute Stokes shift is directly connected to the width of the energy gap distribution within linear response theory ${ }^{80,81}$ via eqn (6). Different absolute shifts $\Delta \Delta U$ therefore correspond to different widths of the distribution of $\Delta U$. As obvious from Table 3 , the studied systems do not show large changes of the width. Within the linear response framework, the width of the energy gap in the ground and excited state cannot differ, so that a change in width is directly connected to a nonlinear response. ${ }^{81,82}$ In ref. 36 we observed furthermore that also large intermediate changes in $W(t)$, a so called intermediate broadening of the spectral width can cause deviations from LRT. Fig. 5 shows the time-evolution of the spectral width obtained from nonequilibrium simulations, as well as the widths in equilibrium.

In contrast to small artificial solutes, which are known to show non-stationary statistics (intermediate broadening), 4,36,83 which correlates with LRT failure, the real chromophores MQ and C153 do not show significant intermediate broadening. There is also no large difference between the spectral widths in the equilibrated states (circles in Fig. 5). The observed failure of LRT can therefore not be attributed to time-dependent changes of the energy gap distribution and has to be sought elsewhere. We furthermore note that the evaluation of the width might be biased, since the sampling of some configurations via a long MD run is statistically hampered, similar to observations of King and Warshel. ${ }^{84}$ For the large chromophores MQ and C153 undergoing only minor changes upon excitation such an effect should be small, but might be non-negligible for smaller artificial solutes, for example in ref. 4 and 36 .

To sum up, from the ten studied systems only MQ and C153 in ACN showed to completely obey LRT, although the interaction energy between solute and solvent is locally Gaussian

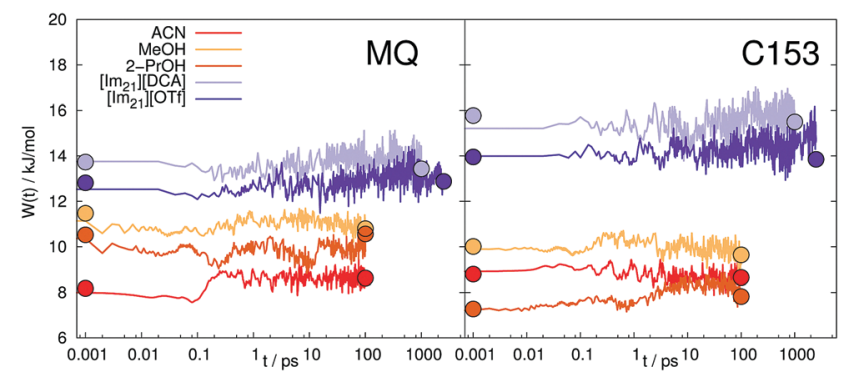

Fig. 5 Time-evolution of the spectral width $W(t)$ of the interaction energy between MQ (left) or C153 (right) and different solvents. The circles at the edges of the curves represent the corresponding widths of the equilibrium simulations. 
and stationary in all systems. LRT mainly fails to describe the inertial solvation response, but also leads to deviations on the timescales of diffusion and rotation. We note that $\mathrm{C} 153$ in 2-PrOH shows only (comparably) small deviations. To further examine the validity of LRT in the polar solvents, we calculated the nonequilibrium response of the deexcitation of the chromophore in $\mathrm{MeOH}$ and 2-PrOH. In the case of linear response, the timescales of solvent relaxation after excitation and deexcitation of a solute should be indistinguishable. The respective relaxation functions are shown in Fig. 1 as dashed curve, labeled $S_{\mathrm{R}}$. For both chromophores, the forward and backward reaction produce different timescale of rearrangement. A lowering of the dipole moment produces for all four solute-solvent combinations a faster solvent rearrangement than a strengthening. In other words, a decrease in order is faster accomplished than an increase in order. The discrepancy between $S(t)$ and $S_{\mathrm{R}}(t)$ is large for $\mathrm{MQ}$, but very small for C153. A closer look at the forward and reverse excitation of $\mathrm{C} 153$ in $\mathrm{MeOH}$ reveals another interesting fact: Although $C_{\mathrm{g}}(t)=C_{\mathrm{e}}(t)$, and $S(t) \simeq$ $S_{\mathrm{R}}(t)$, which is typical for a linear response, ${ }^{3,85}$ the time correlation functions from equilibrium simulations are not capable of describing the correct timescale or curvature of $S(t)$. This effect was also found by ref. 7, and we therefore note that neither $C_{\mathrm{g}}(t)=$ $C_{\mathrm{e}}(t)$ nor $S(t) \simeq S_{\mathrm{R}}(t)$ can give insights on the validity of LRT.

\subsection{Partial correlation functions}

To disentangle the contributions from anions and cations to the overall solvation response, the relaxation function $S(t)$ can easily be separated, as $\Delta U$ is simply a sum of pair interactions, so that

$$
S_{Z}(t)=\frac{\overline{\Delta U_{Z}(t)}-\overline{\Delta U_{Z}(\infty)}}{\overline{\Delta U(0)}-\overline{\Delta U(\infty)}}
$$

where $Z$ can be any contribution, here from cation movement and anion movement. The contributions in equilibrium simulations can be obtained via the partial correlation function

$$
C_{Z}(t)=\frac{\left\langle\delta \Delta U_{Z}(0) \delta \Delta U(t)\right\rangle}{\left\langle\delta \Delta U(0)^{2}\right\rangle}=\frac{\left\langle\delta \Delta U(0) \delta \Delta U_{Z}(t)\right\rangle}{\left\langle\delta \Delta U(0)^{2}\right\rangle}
$$

which directly corresponds to $S_{Z}(t)$. Autocorrelation functions of the different contributions are not comparable to $S_{Z}(t)$, since such a partitioning leads to large cross-correlations. Fig. 6 shows the contributions from cations and anions obtained from nonequilibrium and equilibrium simulations in [ $\left.\operatorname{Im}_{21}\right][\mathrm{OTf}]$. The corresponding figure for $\left[\operatorname{Im}_{21}\right][\mathrm{DCA}]$ can be found in the ESI. $\dagger$ For MQ in the nonequilibrium simulations the anions contribute more to the overall response and their movement occurs on a longer timescale, so that the anionic contribution rises with time. The equilibrium simulations, however, do not depict this behavior. Here, the response is made up one half by anion movement, the other half by cation movement which occurs on the same timescale, and the respective contributions do not change in time. For C153, this discrepancy becomes even larger, as the cationic contribution falls to 0 within $1 \mathrm{ps}$ in the nonequilibrium simulation, but contributes nearly $50 \%$ to the overall Stokes shift on all timescales in the equilibrium

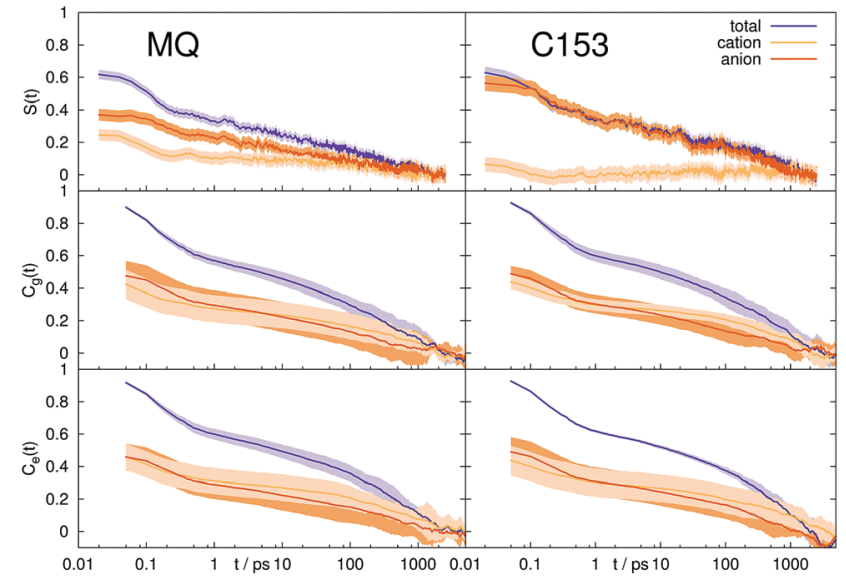

Fig. 6 Contributions from cations and anions to the Stokes shift relaxation function $S(t)$ (top panel) and time correlation functions $C_{g}(t)$ (middle panel) and $C_{e}(t)$ (bottom panel) in ground and excited state in $\left[\mathrm{Im}_{21}\right][\mathrm{OTf}]$ for $\mathrm{MQ}$ (left) and C153 (right). The colored area corresponds to a 95\% confidence interval.

simulation. Here, LRT is clearly not capable of depicting accurate magnitudes of cationic and anionic contributions. A similar phenomenon (a hidden breakdown of linear response), where $S(t)$ and $C(t)$ correspond approximately to each other, but individual contributions do not obey linear response, was already observed by Schwartz and coworkers for rotational and translational contributions to the solvation response. ${ }^{86,87}$

\subsection{Higher-order correlation functions}

Sometimes the applicability of LRT is tested via the validity of eqn (5). ${ }^{35,50,88}$ We thus calculated higher-order correlation functions for all systems up to the order of five, both for the overall response, as well as for the cationic and anionic contributions. The corresponding data is shown in Fig. 7 for $2-\mathrm{PrOH}$ and in the Supporting Information ${ }^{\dagger}$ for all other solvents. Despite deviations from eqn (5) for $\mathrm{MQ}$ in 2-PrOH in the odd order correlation functions, and for $\mathrm{C} 153$ in 2-PrOH in the even order correlation functions, the relation seems to hold for all other systems within the 95\% confidence interval given.

Thus, this analysis of higher-order correlations points towards applicability of LRT for all solvent but 2-PrOH. As we

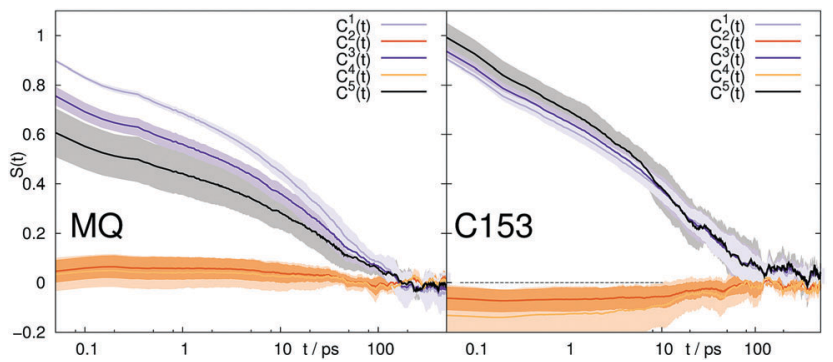

Fig. 7 Higher order correlation functions $C^{n}$, where $C^{n}=\frac{\left\langle\delta \Delta U(0)^{n} \delta \Delta U(t)\right\rangle_{\mathrm{e}}}{a_{n}\left\langle\delta \Delta U(0)^{n+1}\right\rangle_{\mathrm{e}}}$ and $a_{1}=1, a_{2}=1.6, a_{3}=3, a_{4}=6.4$ and $a_{5}=15$ according to eqn (5) after rearrangement. System: 2-PrOH for $\mathrm{MQ}$ (left) and C153 (right). The colored area corresponds to a $95 \%$ confidence interval. 
have seen earlier, LRT fails (i.e. $S(t)$ does not equal $C(t)$ ) for all systems but ACN, so that observing higher-order correlation functions in accordance to eqn (5) does not guarantee validity of the linear response formalism, and should thus be treated very cautiously. Since only the first few higher-order correlations can be calculated with acceptable signal-to-noise ratio and reasonable confidence intervals, we miss any deviations from eqn (5) in all correlation functions of order higher than 5 (odd) or higher than 4 (even). Furthermore, if the equilibrium trajectory does not sample all important configurations, ${ }^{84}$ i.e. ergodicity is not reached, $C(t)$ might not resemble $S(t)$ even if eqn (5) is obeyed.

Recently, corrections to the correlation function via higherorder correlations were suggested for systems were eqn (5) is not fully obeyed. We therefore calculated corrections up to the order of five, via the suggested scheme from ref. 88,

$$
\begin{aligned}
C_{\mathrm{e}}^{\mathrm{corr}} \simeq & \frac{1}{3 k_{\mathrm{B}} T} \sum_{n=0}^{2} \frac{n ! 2^{n}}{(2 n+1) !} \frac{\left\langle\delta \Delta U(0)^{2 n+1} \delta \Delta U(t)\right\rangle_{\mathrm{e}}}{\left\langle\delta \Delta U(0)^{2}\right\rangle_{\mathrm{e}}^{n}} \\
& +\frac{1}{2\left(k_{\mathrm{B}} T\right)^{2}}\left\langle\delta \Delta U(0) \delta \Delta U(t)^{2}\right\rangle_{\mathrm{e}}
\end{aligned}
$$

where the first term can be directly derived from eqn (5), and is a simple average of the first three uneven correlation functions. The first even term (second order) is included via the second term. The inclusion of higher-order correlation functions of order 4 was omitted, as the signal-to-noise ratio was unacceptably low. To normalize this function, it is simply divided by its value at $t=0$. Fig. 8 shows the uncorrected and corrected correlation functions, as well as the nonequilibrium results for both chromophores in 2-PrOH.

For C153 the corrected correlation function comes closer to the true nonequilibrium results, yielding nearly quantitative agreement. This improvement is reasonable, since the even order higher-correlation functions are nonzero and contribute to the observed solvation response. Thus, solvation dynamics of C153 in 2-PrOH can be roughly described within the corrected linear response formalism. However, for all other systems (shown in the ESI $\dagger$ ), the correction does not provide a significant improvement and sometimes even moves the curve in the wrong direction.

Furthermore we calculated higher-order correlation functions for the cation and anion contributions to the overall response

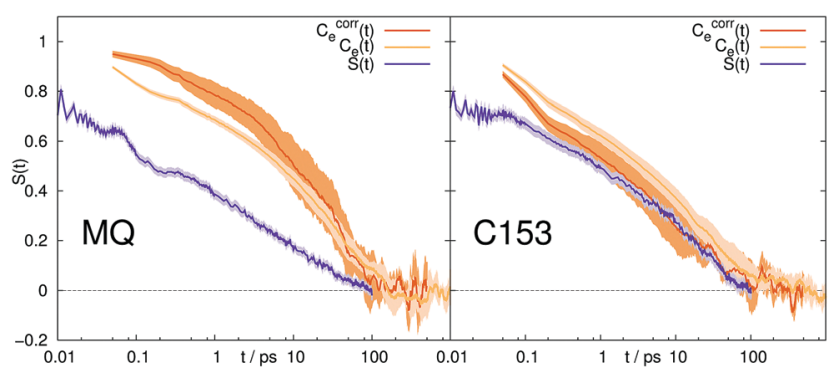

Fig. 8 Normalized correction $C_{e}^{c o r r}$ to the time correlation function $C_{e}(t)$ according to eqn (11) in 2-PrOH for MQ (left) and C153 (right). The colored area corresponds to a $95 \%$ confidence interval. (data shown in the ESI $\dagger$ ). Again, eqn (5) is obeyed within the confidence interval, so that the previously observed failure of LRT for the partial correlation functions could not have been predicted by the partial higher-order correlation functions.

\subsection{Influence of solvent structure}

Whenever the structure of the solvent changes significantly after excitation of the solute, LRT is likely to fail. ${ }^{70-72,89,90} \mathrm{We}$ therefore calculated the radial distribution functions $g(r)$ around MQ and C153 in the ground and excited state in all five solvents, as well as the time evolution of the cation and anion coordination number via Voronoi tessellation. Fig. 9 shows the corresponding results for both chromophores in $\left[\operatorname{Im}_{21}\right][\mathrm{OTf}]$, all other solvents are shown in the ESI. $\dagger$

The excitation of the solute causes slight adaption of the solvent structure in all systems, where MQ inflicts larger structure changes in the surrounding solvent than C153. In polar solvents, this trend can be seen directly from the radial distribution functions, and in ionic liquids from the change in coordination numbers after excitation. For example, after excitation of MQ in [ $\left.\mathrm{Im}_{21}\right][$ OTf] (left panels in Fig. 9), the anion coordination number increases by approximately 0.5 , with only moderate changes in the structure of the closest molecules seen in $g(r)$. The excitation of $\mathrm{C} 153$ in ionic liquids, in contrast, induces larger structure changes of the few innermost ions (change of peak positions and intensities in the radial distribution functions), but the coordination number does not change significantly. Note that the first solvation shell extends to quite large distances, here up to about $8 \AA$. For $\mathrm{MQ}$ in $\mathrm{MeOH}, 2-\mathrm{PrOH},\left[\mathrm{Im}_{21}\right][\mathrm{DCA}]$ and $\left[\mathrm{Im}_{21}\right][\mathrm{OTf}]$, as well as $\mathrm{C} 153$ in $\left[\mathrm{Im}_{21}\right][\mathrm{DCA}]$ and $\left[\mathrm{Im}_{21}\right][\mathrm{OTf}]$ a failure of LRT could be due to rearrangements in the solvent structure visible in the radial distribution functions or ion coordination number. C153 in polar solvents, however, does
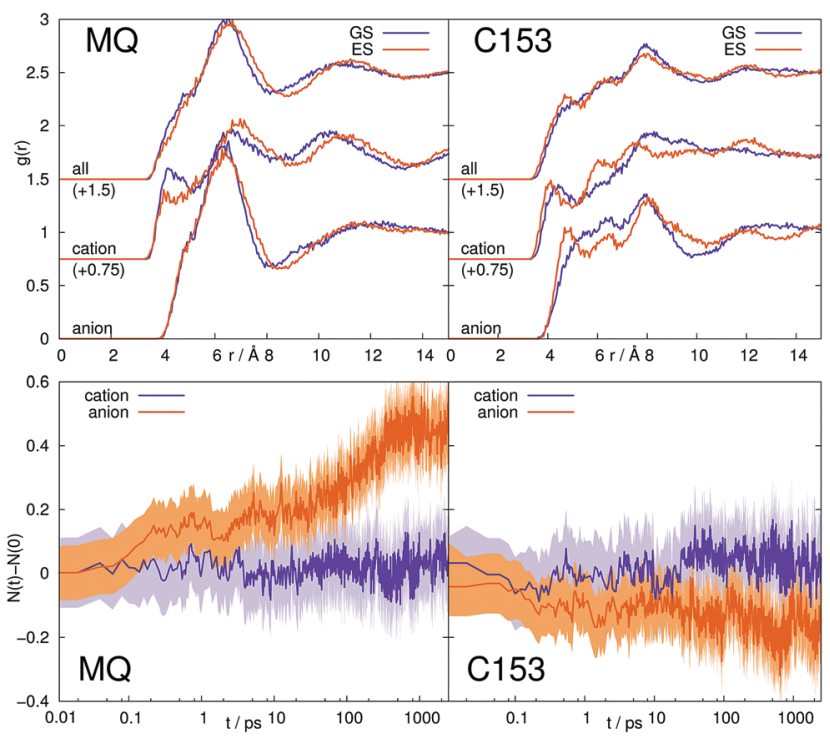

Fig. 9 Top: Radial distribution functions of solvent molecules around the central solute $M Q$ (left), or C153 (right) in [ $\left.\mathrm{Im}_{21}\right]$ [OTf]. Bottom: Change in number of first shell ions around the central solute for the same system. The colored area corresponds to a 95\% confidence interval. 
Table 4 Number of hydrogen bonds in the equilibrated ground and excited state, as well as timescale of the relaxation to the new number of hydrogen bonds after excitation (or deexcitation) of the solute, $\tau_{\text {relax }}$, where applicable

\begin{tabular}{|c|c|c|c|c|}
\hline & \multicolumn{2}{|l|}{ \# of H-bonds } & \multicolumn{2}{|l|}{$\tau_{\text {relax }}[\mathrm{ps}]$} \\
\hline & GS & ES & $\mathrm{GS} \rightarrow \mathrm{ES}$ & $\mathrm{ES} \rightarrow \mathrm{GS}$ \\
\hline \multicolumn{5}{|l|}{ MQ } \\
\hline $\mathrm{MeOH}$ & $2.48 \pm 0.07$ & $1.19 \pm 0.06$ & 5.9 & 19.7 \\
\hline 2-PrOH & $2.72 \pm 0.05$ & $1.58 \pm 0.08$ & 12.2 & 21.5 \\
\hline$\left[\operatorname{Im}_{21}\right][\mathrm{DCA}]$ & $0.017 \pm 0.002$ & $0.005 \pm 0.001$ & - & - \\
\hline$\left[\mathrm{Im}_{21}\right][\mathrm{OTf}]$ & $0.016 \pm 0.006$ & $0.008 \pm 0.002$ & - & - \\
\hline \multicolumn{5}{|l|}{ C153 } \\
\hline $\mathrm{MeOH}$ & $0.77 \pm 0.03$ & $1.36 \pm 0.06$ & 12.3 & 7.8 \\
\hline 2-PrOH & $1.10 \pm 0.04$ & $1.83 \pm 0.04$ & 19.5 & 12.2 \\
\hline$\left[\operatorname{Im}_{21}\right][\mathrm{DCA}]$ & $0.002 \pm 0.001$ & $0.003 \pm 0.001$ & - & - \\
\hline$\left[\operatorname{Im}_{21}\right][$ OTf $]$ & $0.002 \pm 0.001$ & $0.003 \pm 0.001$ & - & - \\
\hline
\end{tabular}

not produce significant changes in structure, so that for these system, validity of LRT is expected, at least from this point of view. The observed failure of the linear response approximation of $\mathrm{C} 153$ in $\mathrm{MeOH}$ is thus not predictable from a structure analysis via radial distribution functions.

We furthermore calculated the number of hydrogen bonds in the equilibrated ground and excited state, as well as the time dependency of the number of hydrogen bonds after excitation of the solute (in nonequilibrium), Table 4. Criterias for hydrogen bonds (maximum distance of $2.2 \AA$, minimum angle of $130 \AA$ ) were taken from Hunt et al. ${ }^{91}$ Possible hydrogen bond donors are the hydroxyl hydrogens in $\mathrm{MeOH}$ and 2-PrOH, as well as the three hydrogens attached to the imidazolium ring in $\left[\mathrm{Im}_{21}\right][\mathrm{DCA}]$ and $\left[\mathrm{Im}_{21}\right][\mathrm{OTf}]$. Possible acceptors are the oxygen atoms in MQ and C153 (no hydrogen bonds were found for the nitrogen atom in C153).

In ionic liquids, hydrogen bonds occur neither in the ground, nor in the excited state of MQ and C153. In $\mathrm{MeOH}$ and 2-PrOH, the number of hydrogen bonds varies between the equilibrated ground and excited state. For example, MQ has on average 2.5 hydrogen bonds in the ground state and eventually loses 1.3 hydrogen bonds after excitation. C153, in contrast increases the number of hydrogen bonds after excitation. The timescale of the decrease or increase in hydrogen bonds after excitation is also given in Table 4. Throughout all systems, a decrease of the number of hydrogen bonds is faster than an increase. The normalized number of hydrogen bonds after excitation is shown in Fig. 10, where the number of hydrogen bonds in the equilibrated ground state (instance of excitation) corresponds to 1 , and in the equilibrated excited state to 0 . For comparison, the timescale of solvent relaxation via the time-dependent Stokes shift is shown, too. Disregarding the inertial part of the response (before $0.1 \mathrm{ps}$ ) the rearrangement of hydrogen bonds occurs approximately on the same timescale as the overall solvent relaxation for MQ. In contrast, the hydrogen bonds after excitation of C153 rearrange slower than the overall solvent relaxation.

A change in number of hydrogen bonds and the corresponding timescale cannot be predicted by equilibrium simulations, so that linear response theory is likely to fail for MQ and C153 in $\mathrm{MeOH}$

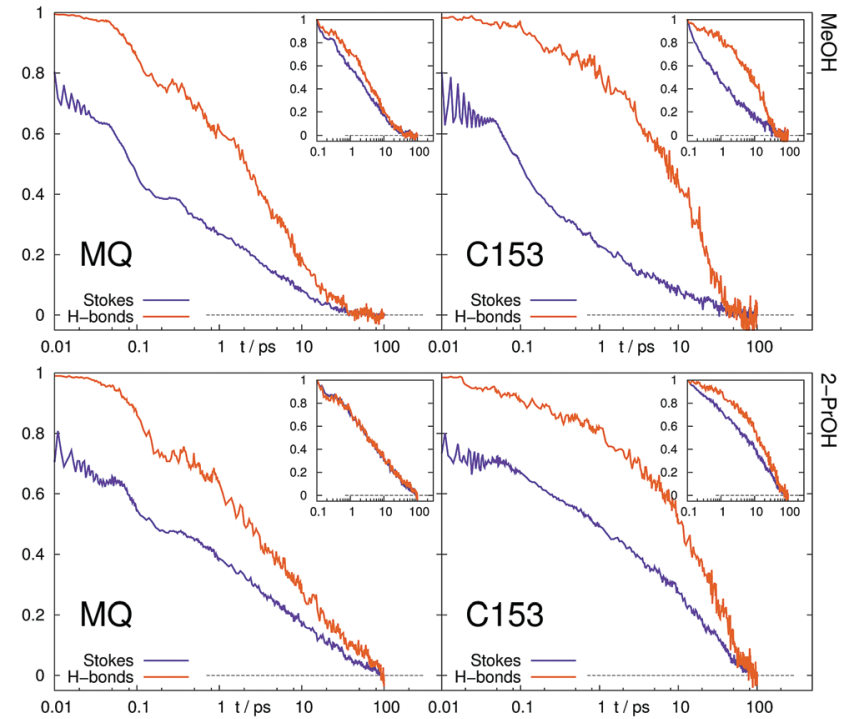

Fig. 10 Time evolution of normalized number of hydrogen bonds compared to the normalized Stokes shift. Inset: Normalization at 0.1 ps.

and 2-PrOH. A nonlinear response associated with different hydrogen bonding patterns in the ground and excited was also previously observed by Ladanyi and coworkers for artificial diatomic solutes in methanol. ${ }^{70-72}$ The observed failure of LRT for $\mathrm{C} 153$ in $\mathrm{MeOH}$ and 2-PrOH is thus plausible, as the hydrogen bonding structure changes after excitation.

\section{Conclusion}

We used simulations of two well known fluorescence probes, MQ and C153, in the polar liquids ACN, MeOH and 2-PrOH, as well as in the ionic liquids $\left[\operatorname{Im}_{21}\right][\mathrm{DCA}]$ and $\left[\mathrm{Im}_{21}\right][\mathrm{OTf}]$ to assess the validity of linear response approximations for different solute-solvent combinations. The solvation response from nonequilibrium simulations perfectly reproduced experimental data for all systems (where experimental data exists), even on an absolute scale. Comparison of equilibrium and nonequilibrium solvation response functions showed that LRT neither yields the correct timescale nor absolute magnitude of the time-dependent Stokes shift for most systems, where LRT fails most severely for the inertial solvation response. A correction to the linear response estimate proposed by Thompson and coworkers ${ }^{88}$ proved to be helpful only in one of all systems studied (C153 in 2-PrOH). Furthermore, the cationic and anionic contributions to the overall shift in the case of ionic liquids could not be reproduced with LRT, yielding quantitatively wrong results.

One should therefore ask whether this failure of LRT could have been predicted from equilibrium simulations. In literature, often the computation of higher-order correlation functions and their factorizations is proposed as a test for LRT validity. ${ }^{35,50,88}$ We found, however, that most of the system studied here easily pass this test, although a comparison of equilibrium and nonequilibrium response curves reveals a severe failure of LRT. Likewise, the magnitude of corrections to the time-correlation 
function via higher-order correlation function cannot be used to predict LRT failure or applicability. Also the existence of a local Gaussian distribution of the energy gap in the ground and excited state, and even its stationarity did not guarantee $C(t) \simeq S(t)$ in all cases studied, i.e. did not prove a global gaussian energy gap. We also found systems, where $C_{\mathrm{e}}(t) \simeq C_{\mathrm{g}}(t)$ and $S(t) \simeq S_{\mathrm{R}}(t)$, that is, a forward and reverse change in solute electron distribution leads to the same response, but $C(t) \neq S(t)$. A good estimate of LRT applicability was found to be the solvent structure in the ground and excited state, i.e. the radial distribution function, coordination number and number of hydrogen bonds. Whenever a large change in solvent structure occurs after excitation, LRT is likely to fail.

For a clear assessment of the validity of LRT in a system the absolute and normalized LRT response function should be compared to experiment or nonequilibrium simulation results. Whenever no experimental data is available for a system, we recommend to calculate the solvent structure in the ground and excited state. If no large changes are found, the time evolution of the spectral width, as well as the absolute solvation response should be calculated from a small number of nonequilibrium simulations and compared to the LRT prediction. Although a small ensemble of nonequilibrium simulations might not yield statistically significant results itself, the magnitude of the shift, the approximate curvature of the response function and changes in the spectral width can easily be compared to the respective results from equilibrium simulations and give a reliable impression of the applicability of LRT. The use of linear response approximations to describe the solvation dynamics of real chromophores in real solvents without a careful testing of its applicability should not be encouraged. We also note that solvation dynamics around real chromophores is a lot more difficult to predict than around simple artificial solutes found in literature. , $35,36,70,88^{-1}$

\section{Conflicts of interest}

There are no conflicts to declare.

\section{Acknowledgements}

This work was partly funded by the Austrian Science Fund FWF in the context of Project No. FWF-P28556-N34. E. H. is recipient of a DOC Fellowship of the Austrian Academy of Sciences at the Institute of Computational Biological Chemistry. The computational results presented have been achieved in part using the Vienna Scientific Cluster (VSC).

\section{References}

1 B. Bagchi, D. W. Oxtoby and G. R. Fleming, Chem. Phys., 1983, 86, 257.

2 M. Maroncelli and G. R. Fleming, J. Chem. Phys., 1987, 86, 6221.

3 M. Maroncelli, J. Chem. Phys., 1991, 94, 2084.

4 E. A. Carter and J. T. Hynes, J. Chem. Phys., 1991, 94, 5961.

5 M. Maroncelli, J. Mol. Liq., 1993, 57, 1.
6 C. F. Chapman, R. S. Fee and M. Maroncelli, J. Phys. Chem., 1995, 99, 4811.

7 M. S. Skaf and B. M. Ladanyi, J. Phys. Chem., 1996, 100, 18258.

8 E. L. Mertz, V. A. Tikhomirov and L. I. Krishtalik, J. Phys. Chem. A, 1997, 101, 3433.

9 M. T. Sonoda, N. H. Moneira, L. Martínez, F. W. Favero, S. M. Vechi, L. R. Martins and M. S. Skaf, Braz. J. Phys., 2004, 34, 3 .

10 J. L. P. Lustres, S. A. Kovalenko, M. Mosquera, T. Senyushkina, W. Flasche and N. P. Ernsting, Angew. Chem., Int. Ed., 2005, 44, 5635.

11 F. Ingrosso, B. M. Ladanyi, B. Mennucci, M. D. Elola and J. Tomasi, J. Phys. Chem. B, 2005, 109, 3553.

12 B. Bagchi and B. Jana, Chem. Soc. Rev., 2010, 39, 1936.

13 R. A. Nome, J. Braz. Chem. Soc., 2010, 21, 2189.

14 M. Sajadi, M. Weinberger, H.-A. Wagenknecht and N. P. Ernsting, Phys. Chem. Chem. Phys., 2011, 13, 17768.

15 C. Allolio, M. Sajadi, N. P. Ernsting and D. Sebastiani, Angew. Chem., Int. Ed., 2013, 52, 1813.

16 A. Petrone, G. Donati, P. Caruso and N. Rega, J. Am. Chem. Soc., 2014, 136, 14866.

17 R. Karmakar and A. Samanta, J. Phys. Chem. A, 2002, 106, 4447. 18 J. A. Ingram, R. S. Moog, N. Ito, R. Biswas and M. Maroncelli, J. Phys. Chem. B, 2003, 107, 5926.

19 S. Sha, P. K. Mandal and A. Samanta, Phys. Chem. Chem. Phys., 2004, 6, 3106.

20 P. K. Mandal, S. Saha, R. Karmakar and A. Samanta, Curr. Sci., 2006, 90, 301.

21 S. Arzhantsev, H. Jin, G. A. Baker and M. Maroncelli, J. Phys. Chem. B, 2007, 111, 4978.

22 H. Jin, G. A. Baker, S. Arzhantsev, J. Dong and M. Maroncelli, J. Phys. Chem. B, 2007, 111, 7291.

23 Y. Shim and H. J. Kim, J. Phys. Chem. B, 2008, 112, 11028.

24 A. Samanta, J. Phys. Chem. Lett., 2010, 1, 1557.

25 D. Roy and M. Maroncelli, J. Phys. Chem. B, 2012, 116, 5951.

26 M. Maroncelli, X.-X. Zhang, M. Liang, D. Roy and N. P. Ernsting, Faraday Discuss., 2012, 154, 409.

27 M. Schmollngruber, C. Schröder and O. Steinhauser, J. Chem. Phys., 2013, 138, 204504.

28 S. Daschakraborty and R. Biswas, J. Chem. Phys., 2013, 139, 164503.

29 Y. Shim and H. J. Kim, J. Phys. Chem. B, 2013, 117, 11743.

30 M. N. Kobrak, J. Chem. Phys., 2006, 125, 064502.

31 Y. Shim, J. Duan, M. Y. Choi and H. J. Kim, J. Chem. Phys., 2003, 119, 6411.

32 Y. Shim, M. Y. Choi and H. J. Kim, J. Chem. Phys., 2005, 122, 044511.

33 R. Zwanzig, Nonequilibrium Statistical Mechanics, Oxford Univ. Press, New York, 2001.

34 B. B. Laird and W. H. Thompson, J. Chem. Phys., 2007, 126, 211104.

35 B. B. Laird and W. H. Thompson, J. Chem. Phys., 2011, 135, 084511.

36 E. Heid, W. Moser and C. Schröder, Phys. Chem. Chem. Phys., 2017, 19, 10940.

37 C. J. Margulis, Mol. Phys., 2004, 102, 829. 
38 B. L. Bhargava and S. Balasubramanian, J. Chem. Phys., 2005, 123, 144505.

39 Y. Shim, D. Jeong, M. Y. Choi and H. J. Kim, J. Chem. Phys., 2006, 125, 061102.

40 M. N. Kobrak, J. Chem. Phys., 2007, 127, 184507.

41 Y. Shim and H. J. Kim, J. Phys. Chem. B, 2007, 111, 4510.

42 Z. L. Terranova and S. A. Corcelli, J. Phys. Chem. B, 2013, 117, 15659.

43 E. C. Wu and H. J. Kim, J. Phys. Chem. B, 2016, 120, 4644.

44 Z. Hu and C. J. Margulis, PNAS, 2006, 103, 831.

45 H. K. Kashyap and R. Biswas, J. Phys. Chem. B, 2010, 114, 254.

46 T. Pal and R. Biswas, J. Chem. Phys., 2014, 141, 104501.

47 P. K. Chowdhury, M. Halder, L. Sanders, T. Calhoun, J. L. Anderson, D. W. Armstrong, X. Song and J. W. Petrich, J. Phys. Chem. B, 2004, 108, 10245.

48 M. N. Kobrak and V. Znamenskiy, Chem. Phys. Lett., 2004, 395, 127.

49 S. Arzhantsev, H. Jin, N. Ito and M. Maroncelli, Chem. Phys. Lett., 2006, 417, 524.

50 T. Li and R. Kumar, J. Chem. Phys., 2015, 143, 174501.

51 G. C. Wick, Phys. Rev., 1950, 80, 268.

52 B. R. Brooks, C. L. Brooks III, A. D. MacKerell Jr., L. Nilsson, R. J. Petrella, B. Roux, Y. Won, G. Archontis, C. Bartels, S. Boresch, A. Caflisch, L. Caves, Q. Cui, A. R. Dinner, M. Feig, S. Fischer, J. Gao, M. Hodoscek, W. Im, K. Kuczera, T. Lazaridis, J. Ma, V. Ovchinnikov, E. Paci, R. W. Pastor, C. B. Post, J. Z. Pu, M. Schaefer, B. Tidor, R. M. Venable, H. L. Woodcock, X. Wu, W. Yang, D. M. York and M. Karplus, J. Comput. Chem., 2009, 30, 1545.

53 E. Heid, S. Harringer and C. Schröder, J. Chem. Phys., 2016, 145, 164506.

54 K. Vanommeslaeghe and A. D. MacKerell Jr., J. Chem. Inf. Model., 2012, 52, 3144.

55 K. Vanommeslaeghe, E. P. Raman and A. D. MacKerell Jr., J. Chem. Inf. Model., 2012, 52, 3155.

56 K. Vanommeslaeghe, E. Hatcher, C. Acharya, S. Kundu, S. Zhong, J. Shim, E. Darian, O. Guvench, P. Lopes, I. Vorobyov and A. D. MacKerell Jr., J. Comput. Chem., 2010, 31, 671.

57 J.-D. Chai and M. Head-Gordon, Phys. Chem. Chem. Phys., 2008, 10, 6615 .

58 C. M. Breneman and K. B. Wiberg, J. Comput. Chem., 1990, 11, 361.

59 J. N. A. Canongia Lopes, M. F. Costa Gomes and A. A. H. Pádua, J. Phys. Chem. B, 2006, 110, 19586.

60 J. N. Canongia Lopes, J. Deschamps and A. A. H. Pádua, J. Phys. Chem. B, 2004, 108, 2038.

61 J. N. Canongia Lopes and A. A. H. Pádua, J. Phys. Chem. B, 2004, 108, 16893.
62 C. E. S. Bernardes, K. Shimizu, J. C. Lopes, P. Marquetand, E. Heid, O. Steinhauser and C. Schröder, Phys. Chem. Chem. Phys., 2016, 18, 1665.

63 G. Kaminski and W. L. Jorgensen, J. Phys. Chem., 1996, 100, 18010. 64 S. Nosé, J. Chem. Phys., 1984, 81, 511.

65 W. G. Hoover, Phys. Rev. A: At., Mol., Opt. Phys., 1985, 31, 1695. 66 L. Martínez, R. Andrade, G. Birgin and J. M. Martínez, J. Comput. Chem., 2009, 30, 2157.

67 N. Michaud-Agrawal, E. J. Denning, T. B. Woolf and

O. Beckstein, J. Comput. Chem., 2011, 32, 2319.

68 M. L. Horng, J. A. Gardecki, A. Papazyan and M. Maroncelli, J. Phys. Chem., 1995, 99, 17311.

69 X.-X. Zhang, M. Liang, N. P. Ernsting and M. Maroncelli, J. Phys. Chem. B, 2013, 117, 4291.

70 T. Fonseca and B. M. Ladanyi, J. Phys. Chem., 1991, 95, 2116.

71 D. K. Phelps, M. J. Weaver and B. M. Ladanyi, J. Chem. Phys., 1993, 176, 575.

72 T. Fonseca and B. M. Ladanyi, J. Mol. Liq., 1994, 60, 1.

73 P. V. Kumar and M. Maroncelli, J. Chem. Phys., 1995, 103, 3038.

74 G. Moumouzias, D. K. Panopoulos and G. Ritzoulis, J. Chem. Eng. Data, 1991, 36, 20.

75 V. G. Komarenko, V. G. Manzheli and A. V. Radtsig, Ukr. Fiz. Zh. (Russ. Ed.), 1967, 12, 681.

76 S. Paez and M. Contreras, J. Chem. Eng. Data, 1989, 34, 455. 77 M. G. Freire, A. R. R. Teles, M. A. A. Rocha, B. Schroder, C. M. S. S. Neves, P. J. Carvalho, D. V. Evtuguin, L. M. N. B. F. Santos and J. A. P. Coutinho, J. Chem. Eng. Data, 2011, 56, 4813. 78 E. Heid and C. Schröder, J. Phys. Chem. B, 2017, 121, 9639. 79 M. Gerecke, C. Richter, M. Quick, I. Ioffe, R. Mahrwald, S. A. Kovalenko and N. P. Ernsting, J. Phys. Chem. B, 2017, 121, 9631. 80 T. Li, J. Phys. Chem. B, 2014, 118, 12952.

81 M. D. Stephens, J. G. Saven and S. L. Skinner, J. Chem. Phys., 1997, 106, 2129.

82 M. Tachiya, J. Phys. Chem., 1989, 93, 7050.

83 P. L. Geissler and D. Chandler, J. Chem. Phys., 2000, 113, 9759.

84 G. King and A. Warshel, J. Chem. Phys, 1990, 93, 8682.

85 L. Turi, P. Mináry and P. J. Rossky, Chem. Phys. Lett., 2000, 316, 465.

86 M. J. Bedard-Hearn, R. E. Larsen and B. J. Schwartz, J. Phys. Chem. A, 2003, 107, 4773.

87 M. J. Bedard-Hearn, R. E. Larsen and B. J. Schwartz, J. Phys. Chem. B, 2003, 107, 14464.

88 A. J. Schile and W. H. Thompson, J. Chem. Phys., 2017, 146, 154109.

89 A. C. Moskun, A. E. Jailaubekov, S. E. Bradforth, G. Tao and R. M. Stratt, Science, 2006, 311, 1907.

90 G. Tao and R. M. Stratt, J. Chem. Phys., 2006, 125, 114501.

91 P. A. Hunt, C. R. Ashworth and R. P. Matthews, Chem. Soc. Rev., 2015, 44, 1257. 